\title{
Mycobacterial culture results of smear-positive patients with suspected pulmonary tuberculosis in Liverpool
}

\author{
J.A. Corless*, P.A. Stockton ${ }^{+}$, P.D.O. Davies ${ }^{+}$
}

\begin{abstract}
Mycobacterial culture results of smear-positive patients with suspected pulmonary tuberculosis in Liverpool. J.A. Corless, P.A. Stockton, P.D.O. Davies. (C)ERS Journals Ltd 2000.

ABSTRACT: The aim of this study was to assess the final mycobacterial culture results of patients with smear-positive sputum or bronchial washings and to investigate the efficiency of local tuberculosis (TB) contact-tracing.

Retrospective analysis of mycobacterial cultures and contact-tracing was performed in every patient with smear-positive sputum or bronchoalveolar lavage (BAL) in two Liverpool teaching hospitals (1996-1998). Of these patients 116 with smear-positive sputum or BAL were identified. Mycobacterium tuberculosis (M. tuberculosis) was cultured in $57(49 \%)$, environmental mycobacteria in $37(32 \%)$ and cultures were negative in $22(19 \%)$ of the patients. Contact-tracing information was available in 107 of the $116(92 \%)$ patients. A total number of 1,357 contacts were screened for possible tuberculosis. Of these, $420(31 \%)$ were contacts of patients who cultured environmental organisms or had negative cultures.

In this study, $51 \%$ of smear-positive patients in Liverpool did not have tuberculosis. Inefficiencies in current contact-tracing procedures have been identified which result from screening contacts of index cases that are subsequently found not to have cultured Mycobacterium tuberculosis. The authors believe that there are clear grounds for using rapid tests to identify and type mycobacteria more quickly than current solid or liquid media methods. It is also suggested that regional variations in the frequency of infection with environmental mycobacteria should be considered when formulating tuberculosis contact-tracing procedures.

Eur Respir J 2000; 16: 976-979.
\end{abstract}

\begin{abstract}
*Aintree Chest Centre, University Hospital, Aintree, Lower Lane, Liverpool.

Tuberculosis Research Unit, The Cardiothoracic Centre, Thomas Drive, Liverpool, UK.
\end{abstract}

Correspondence: P.D.O. Davies

Tuberculosis Research Centre

The Cardiothoracic Centre

Thomas Drive

Liverpool L4 3PE

UK

Fax: 441512287688

Keywords: Contact-tracing environmental mycobacteria tuberculosis

Received: April 62000

Accepted after revision July 132000
The aim of contact-tracing is to prevent the spread of tuberculosis. Current contact-tracing procedures in the UK [1-3] rely on early notification of patients with suspected tuberculosis, usually on the basis of a positive sputum smear or bronchoalveolar lavage (BAL) fluid. Following notification of a case of suspected tuberculosis to the "Proper Officer" of the local authority, a program of screening close contacts of the index case is implemented. The tuberculosis (TB) health visitor responsible for the locality in which the index case lives is notified and the index case is then interviewed. The health visitor will then approach each potential contact who will usually be seen in the TB screening clinic at a local hospital. Typically the contact has a chest radiograph and if indicated, a Heaf test. As standard mycobacterial culture techniques may take more than six weeks [4], contacttracing procedures may be undertaken before mycobacterial species and antibiotic sensitivities in the index case are known.

Environmental mycobacteria, unlike Mycobacterium tuberculosis ( $M$. tuberculosis) are ubiquitous organisms that live in media such as water and soil and except in very rare cases do not spread from person to person. Screening of contacts of patients with pulmonary infection from environmental mycobacteria is therefore, unnecessary. In regions with a high incidence of lung disease caused by environmental mycobacteria, the tuberculosis contact- tracing service may theoretically become inefficient as contacts are screened of "index cases" who are later found not to have cultured M. tuberculosis.

No recently published data on the national incidence of respiratory disease due to environmental mycobacteria is available. The authors subjective experience was that despite Liverpool having a similar incidence of tuberculosis to the national average (10.3 notifications 100,000 cases $^{-1}$ in North West England in 1998. The national average for England and Wales being 11.6.100,000 $\mathrm{cases}^{-1}$; source: Public Health Laboratory Service), there also seemed to be a high incidence of environmental mycobacteria. In this study the frequency of pulmonary infections with environmental mycobacteria was investigated in two Liverpool hospitals and the efficiency of the tuberculosis contact-tracing service assessed.

\section{Materials and methods}

The study was carried out at two large teaching hospitals in Liverpool, each with its own independent Microbiology laboratory. Data was collected retrospectively, over a two-year period, on all patients who had positive smears of sputum or bronchoalveolar lavage fluid using an auramine-phenol stain. The final mycobacterial culture results for each patient were then obtained. The first hospital studied, "Hospital A" (Royal Liverpool University 
Hospital, Liverpool, UK) has approximately 800 beds and serves a population of 300,000 people. Data were collected April 1996-April 1998. The second hospital studied, "Hospital B" (University Hospital Aintree, Liverpool, UK) has approximately 900 beds and serves a population of 400,000 people. Data at hospital B were collected February 1996-March 1999. Patients with smear-negative culturepositive tuberculosis or suspected nonpulmonary tuberculosis were not considered. The catchment area of the two hospitals covered central Liverpool and some of its suburbs.

The aim of the second phase of this study was to establish what had happened, in terms of contact-tracing in each case of suspected pulmonary tuberculosis. This was achieved by liaising with every local TB health visitor. A list of names of all the smear-positive patients was provided and the TB health visitors were asked to provide details of the numbers and names of contacts that they had seen in the TB contact clinic.

As the authors source of patient data was the Microbiology databases, only details entered on the initial laboratory request form concerning age, sex and ethnicity were available.

\section{Statistical methods used}

Results of the mycobacterial cultures at the two hospital sites and differences in the mycobacterial cultures between sputum and BAL were analysed using the chi-squared test. Differences between the number of contacts screened for index cases who cultured M. tuberculosis and the group who either cultured environmental mycobacteria or had negative cultures were assessed using the Mann-Whitney U-test.

\section{Results}

A total of 116 "smear-positive" patients were identified, 95 from sputum samples and 21 from BAL fluid. Many patients had multiple samples sent to the laboratory. Fourty-nine patients were from hospital A and 67 patients were from hospital B. A complete breakdown of the final mycobacterial culture results is given in table 1 . This data is summarized in figure 1 which shows that $M$. tuberculosis was cultured in 57 patients (49\%), environmental mycobacteria were cultured in $37(32 \%)$ and cultures were negative in $22(19 \%)$ patients. The proportion of patients in each of the three groups was similar at both hospitals with no statistically significant difference seen. Of the group from whom $M$. tuberculosis was cultured, $42(74 \%)$ were male, $11(19 \%)$ were female and the authors were unable to confirm the sex in 4 (7\%) of the cases. In the group from whom environmental mycobacteria was cultured, $23(62 \%)$ were male and 14 $(38 \%)$ were female. In the smear-positive culture-negative group 14 (64\%) were male, seven (32\%) were female and one $(4 \%)$ was of unknown sex. Details of age were obtained in 62 of 116 patients. Median age for the $M$. tuberculosis group was 47 (range 16-86, $\mathrm{n}=34$ ) and for the non-M. tuberculosis group was 61.5 (range 35-87, $\mathrm{n}=28$ ). Analysis of the patients names suggested that 105 of 116 were of Western European ethnic origin and 11 of 116 were of other ethnic origin.
Table 1. - Final mycobacterial cuture results in 116 patients with positive smears

\begin{tabular}{lrcc}
\hline Culture result & All & Hospital A & Hospital B \\
\hline Subjects n & 116 & 49 & 67 \\
MTB & 57 & 24 & 33 \\
Environmental & & & \\
$\quad$ All species & 37 & 13 & 24 \\
Mycobacteria & & & \\
M. Avium intracellulare & 17 & 8 & 9 \\
M. malmoense & 10 & 2 & 8 \\
M. kansasii & 7 & 3 & 4 \\
M. xenopi & 1 & 0 & 1 \\
M. gordonae & 1 & 0 & 1 \\
M. chelonae & 1 & 0 & 1 \\
Negative & 22 & 12 & 10 \\
\hline
\end{tabular}

MTB: Mycobacterium tuberculosis.

The breakdown of the different environmental mycobacteria that were cultured is given in table 1. Only one of the patients who cultured $M$. avium-intracellulare was known to be HIV-positive.

Statistically significant differences were found in the culture results from BAL compared with sputum $(\mathrm{p}=$ 0.042 ). Mycobacterial cultures from the sputum samples $(\mathrm{n}=95)$ grew $M$. tuberculosis in $50(53 \%)$, environmental mycobacteria in $31(33 \%)$ and were negative in $14(14 \%)$ cases. Cultures from BAL samples $(n=21)$ grew $M$. tuberculosis in $7(33 \%)$, environmental organisms in 6 $(29 \%)$ and were negative in $8(38 \%)$ cases. The BAL samples included a case of $M$. gordonae and M. chelonae which are usually regarded as contaminants.

The details of the 22 patients who were smear-positive and culture-negative were analysed. In 11 of them only a single sputum sample and in nine only a single BAL sample was received by the laboratory (this contrasted with culture-positive patients in whom multiple samples were typically received). In two of the patients multiple sputum samples sent on different days were all smear-positive culture-negative. There was no clustering of the occurrence of smear-positive culture-negative samples around any particular date in either laboratory. Twenty of the patients had not received recent antituberculous therapy. Two patients had previously completed chemotherapy for $M$. tuberculosis and when they represented were found not to have active tuberculosis.

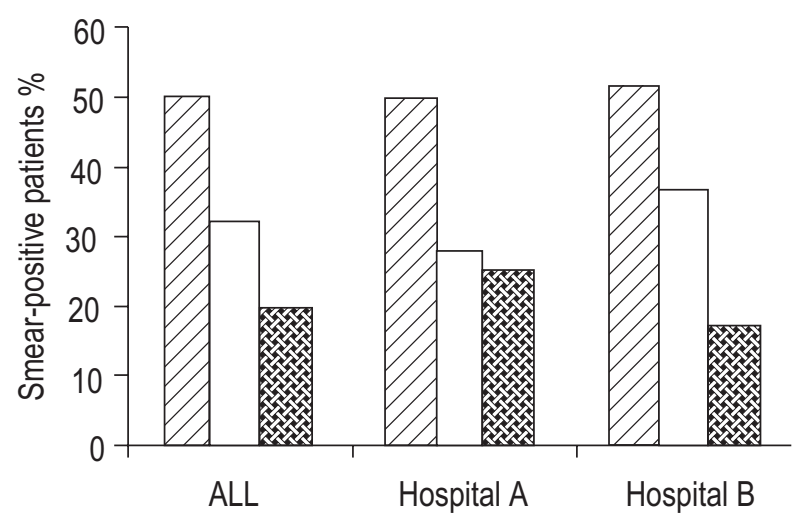

Fig. 1. - Percentage of smear-positive patients culturing Mycobacterium tuberculosis environmental mycobacteria and negative cultures with a breakdown of figures for the two hospitals. (ש): MTB (Mycobacterium tuberculosis); ( $\square$ ) : environmental; (网) : culture-negative. 
The authors were able to establish what had happened with regards to contact-tracing in 107 of 116 cases (92\%). The remaining nine cases were either patients with incomplete demographic data on the initial laboratory request form or patients who lived outside Liverpool and were dealt with by TB health visitors from outside the region. A total of 97 of the remaining 107 patients were initially notified as having suspected tuberculosis. The remaining ten were never notified. Specimens from one of these patients subsequently cultured $M$. tuberculosis with the other nine culturing either environmental organisms or having negative cultures.

A total of 1,357 contacts of 97 patients were screened for possible tuberculosis. Of these 937 (69\%) were contacts of patients who subsequently cultured $M$. tuberculosis and $420(31 \%)$ were contacts of patients who either cultured environmental organisms or who had negative cultures. Therefore, $31 \%$ of all contact-tracing work was unnecessary. A comparison of the number of contacts screened for each index case showed statistically significant differences $(\mathrm{p}=0.004)$ when comparing the patient from whom $M$. tuberculosis was cultured with the group culturing environmental organisms or with negative cultures. The median number of contacts traced.index case ${ }^{-1}$ was 11 (range $0-124$ ) for patients culturing M. tuberculosis and 4 (range $0-47$ ) for patients culturing environmental organisms or with negative cultures. No contact in this study developed tuberculosis while under surveillance.

\section{Discussion}

More than half of all patients in this study who had a positive smear of sputum or BAL did not in fact have tuberculosis. The relatively high frequency of both environmental mycobacteria and negative cultures also resulted in a large amount of unnecessary work for the tuberculosis contact-tracing service. It has been demonstrated that nearly one-third of all contact-tracing work studied in this survey was unnecessary.

The relative fraction of false-positive smear results is defined as the proportion of positive smear results that are culture-negative. It is generally regarded that this figure should be nearer to $5 \%$ than the $19 \%$ and $24 \%$ that is reported in this study, however, there is no recently published data from the UK to support this assumption. A number of older studies exist which demonstrate a wide variation in the relative fraction of smear-positive culturenegative results as outlined in table 2, although not all were limited to respiratory specimens. Potential causes

Table 2. - Percentages of smear-positive culturenegative specimens in previous studies

\begin{tabular}{|c|c|}
\hline Investigators & Total false-positive smears $\%$ \\
\hline BOYD and MARR [5] & 55 \\
\hline NARAIN et al. [6] & 40 \\
\hline TRUANT et al. [7] & 31 \\
\hline Messa et al. [8] & 11 \\
\hline Mitchison et al. [9] & 8 \\
\hline DomingUEZ et al. [10] & 6.5 \\
\hline CHAN et al. [11] & 6 \\
\hline GORDIN et al. [12] & 0.7 \\
\hline BORCHARDT et al. [13] & 0.3 \\
\hline
\end{tabular}

for smear-positive culture-negative specimens include contamination at the time of bronchoscopy, contamination of specimens [14] or inadvertent killing of live mycobacteria in the laboratory [15] and samples sent from patients already started on antituberculous therapy, (two patients with false-positive smears in the present study had previously completed antituberculosis chemotherapy. It was presumed that they were still shedding dead mycobacteria at the time the samples were taken). The fact that the results from two independent hospital laboratories were similar does suggest that this situation is not unique and the causes are probably multifactorial.

The current system of tuberculosis contact-tracing in the UK was developed at a time when the prevalence of tuberculosis was higher than it is today and when environmental organisms were either less common or less well recognized. Even the current UK guidelines for control and prevention of tuberculosis [16, 17] do not mention the potential pitfall caused by notification of suspected tuberculosis, which is subsequently found to be nontuberculous disease. Separate guidelines for notification and management of opportunistic mycobacterial infection [3, 18] discuss "denotification" of patients on the basis of culture results.

The results of the present study suggest that a reevaluation of contact-tracing procedures should be considered. A number of new diagnostic tests are commercially available that can identify and type mycobacteria more quickly than standard solid or liquid culture methods [1924]. It is now possible to determine whether or not a smear-positive sample of sputum or BAL results from infection with $M$. tuberculosis in as little as $24 \mathrm{~h}$. Although these tests have not yet been proven to be totally reliable, they may in the very near future allow for a significant reduction in the standard six week delay for mycobacterial culture results and therefore, eliminate the unnecessary contact-tracing work that results. The extra costs of testing would certainly be offset by, savings in the tuberculosis health visitors and doctors time, a reduction in unnecessary chest radiography and in some cases unnecessary tuberculosis treatment. The psychosocial impact of a false diagnosis of tuberculosis is also an important factor.

To conclude, the authors believe that they have identified local inefficiencies in current contact-tracing procedures, mainly due to an increase in the number of respiratory infections recognized as being caused by environmental mycobacteria. It is suggested that regional variations in the incidence of infection with environmental mycobacteria should be considered when formulating tuberculosis contact-tracing procedures.

\footnotetext{
Acknowledgements. The authors would like to thank all of the tuberculosis health visitors who helped to collate the results for this study and the staff of the two hospital Microbiology departments.
}

\section{References}

1. Notification of tuberculosis: a code of practice for England and Wales. Joint tuberculosis committee of the British Thoracic Association. BMJ 1982; 284: $1454-$ 1456. 
2. Health Services and Public Health Act 1968 c. 46. London, HMSO, 1968; p.36.

3. Ormerod LP, Watson JM, Pozniak A, Kumar D, McManus T. Notification of tuberculosis: an updated code of practice in England and Wales. $J R$ Coll Physicians 1997; 31: 299-303.

4. Drobniewski FA, Watt B, Smith EG, et al. A national audit of the laboratory diagnosis of tuberculosis and other mycobacterial diseases within the United Kingdom. $J$ Clin Pathol 1999; 52: 334-337.

5. Boyd JC, Marr JJ. Decreasing reliability of acid-fast smear techniques for detection of tuberculosis. Ann Intern Med 1975; 82: 489-492.

6. Narain R, Rao MS, Chandrasekhar P, et al. Microscopy positive and microscopy negative cases of pulmonary tuberculosis. Am Rev Respir Dis 1971; 103: 761-773.

7. Truant JP, Brett WA, Thomas W. Fluorescence microscopy of tubercle bacilli stained with auramine and rhodamine. Henry Ford Hosp Med Bull 1962; 10: 287-296.

8. Messa CJ, Blair EB, Tull AH, Andres DH. Computer files and analyses of laboratory data from tuberculous patients. Am Rev Respir Dis 1973; 108: 813-818.

9. Mitchison RS, Allen BW, Mitchison DA. False-positive acid-fast smears. Lancet 1975; 2(7928): 281 [letter].

10. Dominguez JM, Vivas ES. Smear-positive and culture negative results of routine sputum investigations for the detection and therapy control of pulmonary tuberculosis. Tubercle 1977; 58: 217-220.

11. Chan W, Chia A, Lee LK, McFadyen DM. Bacteriolgical measures for the detection of cases of pulmonary tuberculosis. Bull WHO 1971; 45: 551-558.

12. Gordin F, Slutking G. The validity of acid-fast smears in the diagnosis of pulmonary tuberculosis. Arch Pathol Lab Med 1990; 114: 1025-1027.

13. Borchardt KA, Gelber R, Botch V. Acid-fast smears in tuberculosis detection. Ann Intern Med 1976; 84: 344 [letter].

14. Van Deun A, Roorda FA, Chambugonj N, Hye MA, Hossain MA. Reproducibility of sputum smear examination for acid-fast bacilli: practical problems met during cross-checking. Int J Tuberc Lung Dis 1999; 3: 823-829.
15. Van Deun A, Portaels F. Limitations and requirements for quality control of sputum smear microscopy for acid-fast bacilli. Int J Tuberc Lung Dis 1999; 2: 756-765.

16. Joint tuberculosis committee of the British Thoracic Society. Control and prevention of tuberculosis in the United Kingdom: Code of Practice 1994. Thorax 1994; 49: $1193-1200$.

17. Joint tuberculosis committee of the British Thoracic Society. Chemotherapy and management of tuberculosis in the United Kingdom: recommendations 1998. Thorax 1998; 53: 536-548.

18. Subcommittee of the Joint Tuberculosis Committee of the British Thoracic Society. Management of opportunistic mycobacterial infections: Joint tuberculosis committee guidelines 1999. Thorax 2000; 55: 210-218.

19. Woods GL. Molecular methods in the detection and identification of mycobacterial infections. Arch Pathol Lab Med 1999; 123: 1002-1006.

20. Alcaide F, Benitez, MA, Escriba JM, Martin R. Evaluation of the BACTEC MGIT 960 and the MB/ BacT systems for recovery of mycobacteria from clinical specimens and for species identification by DNA AccuProbe. J Clin Microbiol 2000; 38: 398-401.

21. Ruiz-Serrano MJ, Albadalejo J, Martinez-Sanchez L, Bouza E. LCx: a diagnostic alternative for the early detection of Mycobacterium tuberculosis complex. Diagn Microbiol Infect Dis 1998; 32: 259-264.

22. Sion C, Degraux J, Delmee M. Early identification of Mycobacterium tuberculosis and Mycobacterium avium using the polymerase chain reaction on samples positive by a rapid commercial culture system. Eur J Clin Microbiol Infect Dis 1999; 18: 346-351.

23. Hernandez SM, Morlock GP, Butler WR, Crawford JT, Cooksey RC. Identification of Mycobacterium species by PCR-restriction fragment length polymorphism analyses using fluorescence capillary electrophoresis. J Clin Microbiol 1999; 37: 3688-3692.

24. Ryang DW, Ryang DH, Shin MG, Shin JH, Kee SJ, Suh SP. Alternative use of polymerase chain reaction instead of rho-nitro-alpha-acetylamino-beta-hydroxyproplophenone test for the early detection of Mycobacterium tuberculosis in BACTEC 12B cultures. APMIS 1996; 104: 444-450. 